\title{
A Comparison of the Potential Capability of SFS, SPS and HVSFS for the Production of Photocatalytic Titania Coatings
}

\author{
B. Robinson a, A. Tabeckia, S. Paula, G. Shia, A. Mills ${ }^{b}$, I. P. Parkinc, J. A. Darrc and H. L. de Villiers Lovelock \\ aTWI Granta Park, Great Abington, Cambridge, CB21 6AL, UK \\ bSchool of Chemistry and Chemical Engineering, Queen's University Belfast, Belfast, BT9 5AG, UK \\ cDepartment of Chemistry, University College London, 20 Gordon Street, London, WC1H OAJ, UK
}

The photocatalytic capabilities of titanium dioxide are widely published. Reported applications of titania coatings include air purification, water purification and self-cleaning. Suspension spray has been highlighted as a possible route for the deposition of highly active nanostructured $\mathrm{TiO}_{2}$ coatings. Published work has demonstrated the capabilities of suspension plasma spray (SPS) and high velocity suspension flame spray (HVSFS), however, little work exists for suspension flame spray (SFS). Herein, these three suspension spray processes are compared as regards their capability to produce photocatalytic $\mathrm{TiO}_{2}$ coatings and their potential for industrial scale up. A range of coatings were produced for each process, manipulating coating parameters in order to vary phase composition and other coating characteristics to effect activity. Coatings produced were found to vary significantly between the processes with SFS highlighted as the most effective technique with regards to future scale-up and coating photoactivity. SFS coatings were found to be up to 5 times more active than analogous coating produced by CVD.

1

\section{Introduction}

There are currently three suspension spray techniques reported in the literature which have been used to produce photocatalytic titania coatings; suspension plasma spray (SPS), suspension flame spray (SFS) and high velocity suspension flame spray (HVSFS).[1-3] Each of these techniques utilises different thermal spray torches, giving rise to different particle temperatures and velocities.[4] This has significant effects on coating microstructure and phase composition, which in turn affects coating photoactivity. There have been a number of papers which compare the photocatalytic properties of HVSFS and SPS $\mathrm{TiO}_{2}$ coatings.[5] In all instances, SPS has been demonstrated as producing significantly more photoactive $\mathrm{TiO}_{2}$ coatings than the HVSFS process. This is typically attributed to greater retention of the anatase phase in SPS coatings, due to the slower velocity of the jet, which allows resolidification of particles in flight (which recrystallize as anatase phase).[6] However, there are only a limited number of examples reported where photoactive SFS titania coatings have been produced, and there are no examples where they have been compared directly with SPS and HVSFS.[2] Herein, the viability of the three above mentioned suspension spray techniques for producing photoactive titania coatings is assessed. Coatings were characterised by a number of methods including XRD, SEM, UV-Vis, and a sacrificial photocatalytic hydrogen production test. The practical aspects of each of the techniques and which has the greatest potential for future industrial up-scale are also discussed.

\section{Experimental}

\subsection{Spraying Processes}

Suspension Flame Spraying (SFS) was performed using a Metco Flame 5P spray gun with external air atomised suspension injection, Fig $1 \mathrm{~A}$. The atomiser was constructed by modifying a commercial air brush. Air was used as the atomisation gas and acetylene as the fuel gas. The suspension was gravity-fed from a torch mounted reservoir at a rate of approximately $7 \mathrm{~mL} \mathrm{~min}-1$. Oscillations in feed rate of up to $1 \mathrm{~mL} \mathrm{~min}^{-1}$ were observed. During spraying the flame spray torch was mounted on a 6-axis robot for manipulation. SFS spray parameters used listed in Table 1.

Table 1 SFS process parameters.

\begin{tabular}{cc}
\hline Parameter & Value \\
\hline Robot travel velocity / $\mathrm{mms}^{-1}$ & - \\
Increment / $\mathrm{mm}$ & - \\
Acetylene flow rate / FMR & - \\
Oxygen flow rate / FMR & - \\
Number of passes & - \\
Stand-off distance / $\mathrm{mm}$ & - \\
Suspension feed rate / $\mathrm{mL} \mathrm{min}^{-1}$ & - \\
Air atomisation gas / $\mathrm{L} \mathrm{min}^{-1}$ & - \\
\hline
\end{tabular}



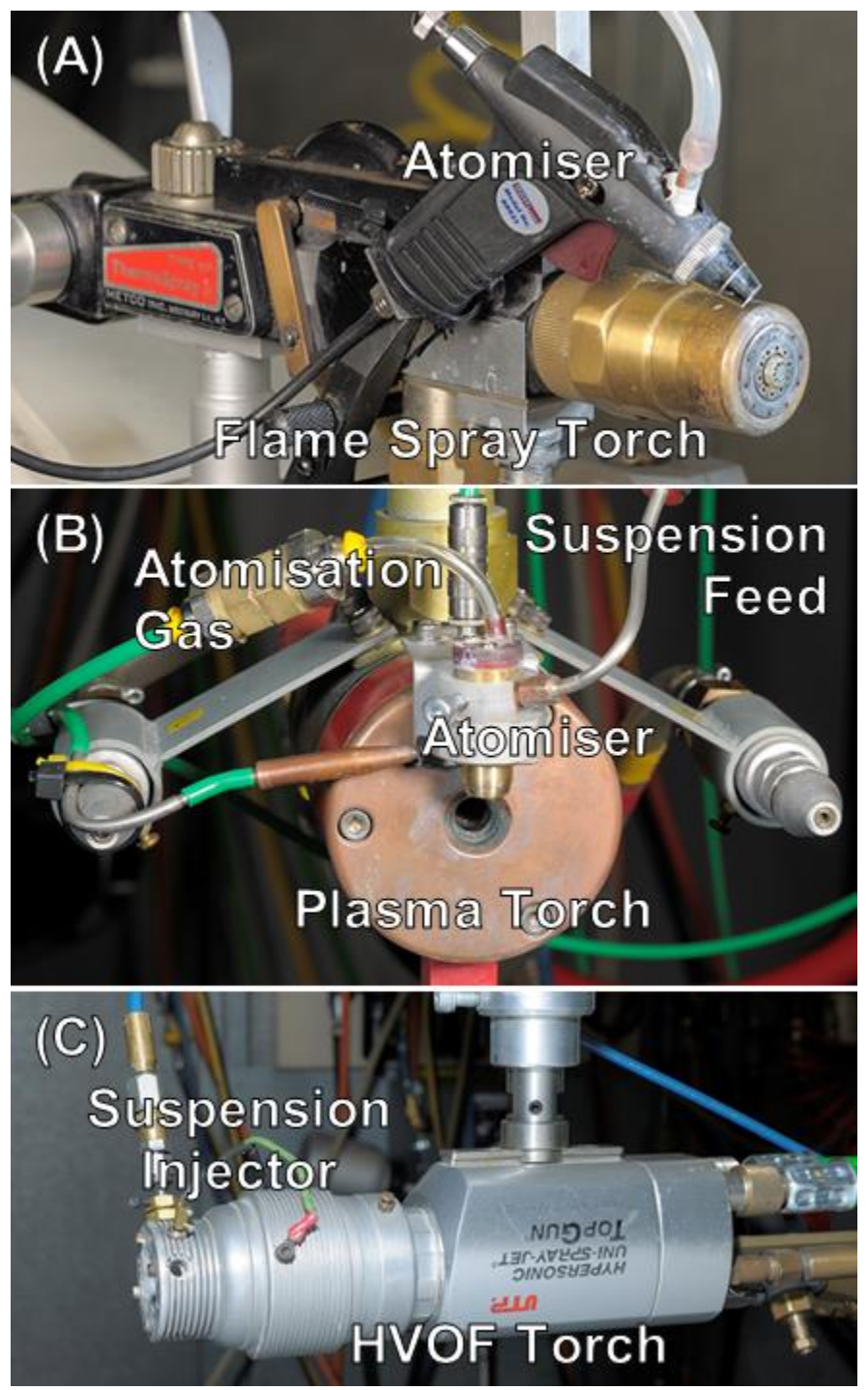

Figure 1 Photograph of the three suspension spray systems, SFS (A), SPS (B) and HVSFS (C).

Suspension plasma spraying (SPS) was performed using a Praxair ${ }^{\circledR S G}-100$ torch, Fig. 1B. Argon and argon/hydrogen mixtures were used as the plasma gases. The nanoparticle suspension was fed to the external atomiser at a flow rate of $5 \mathrm{~mL} \mathrm{~min}^{-1}$ using an ISCO 260D syringe pump (Fig 2B). SPS spray parameters are listed in Table 2. High velocity suspension spraying (HVSFS) was performed using a Top Gun® HVOF torch with propylene fuel, Fig. 1C. Suspension was fed using the same syringe pump through a $0.3 \mathrm{~mm}$ injector nozzle at a flow rate of $20 \mathrm{~mL} \mathrm{~min}^{-1}$. HVSFS spray parameters are listed in Table 2.

Table 2 SPS parameters.

\begin{tabular}{lcc}
\hline \multirow{2}{*}{ Spray Parameter } & \multicolumn{2}{c}{ Parameter Sets } \\
\cline { 2 - 3 } & $\mathbf{A r} / \mathbf{H}_{2}$ & $\mathbf{A r}$ \\
\hline Ar plasma gas flow rate / $\mathrm{L} \mathrm{min}^{-1}$ & 49 & 49 \\
$\mathrm{H}_{2}$ plasma gas flow rate / L min & -1 & -9 \\
Plasma Voltage / V & 45 & 25 \\
Plasma Current / A & 700 & 450 \\
Argon atomisation gas flow rate / & 3 & 3 \\
Number of spray passes & 10 & 10 \\
Spray Distance / mm & $55-85$ & $55-85$ \\
\hline
\end{tabular}


Table 3 HVSFS spray parameters used.

\begin{tabular}{cc}
\hline Spray Parameter & Value \\
\hline Fuel gas pressure / SLPM & 280 \\
Oxygen pressure / SLPM & 80 \\
Number of passes & 5 \\
Increment / mm & 2.5 \\
Stand-off distance / mm & $100-130$ \\
Suspension feed rate / $\mathrm{mLmin}^{-1}$ & 20
\end{tabular}

For all suspension spray trials, samples were water cooled to a temperature of approximately $55^{\circ} \mathrm{C}$ during spraying, using a custom built jig. Water cooling was used to prevent overheating and distortion of the thin ( $0.5 \mathrm{~mm}$ ) substrates.

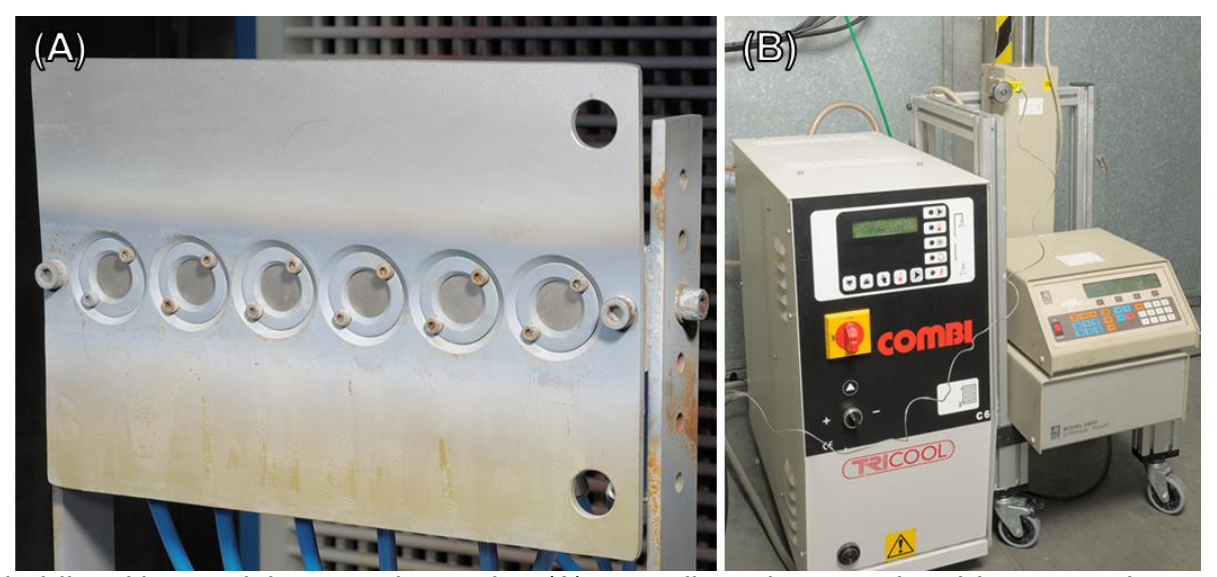

Figure 2 Water cooled jig with 6 stainless steel samples (A) as well as the associated heater exchanger pump (B) and the syringe pump used to feed suspension during SPS and HVSFS trials (B

\section{$2.2 \quad$ Materials}

All suspension spray trials were performed using both 6 and $12 \mathrm{~nm}$ titania suspensions (6.2 $\pm 2.2 \mathrm{~nm}$ and $11.8 \pm 1.8 \mathrm{~nm}$ primary crystallite size, agglomerate size of approximately $20 \mathrm{~nm}$ and $40 \mathrm{~nm}$ respectively) synthesised via continuous hydrothermal synthesis (CHFS), Fig. 3.[7] In CHFS a stream of supercritical water contacts a stream of metal salts of ambient temperature instigating nucleation of metal oxide nanoparticles due to instantaneous hydrolysis and dehydration reactions (Table 4). The reaction was facilitated by the use of a patented confined jet-mixer which controls the mixing of reactants and nucleation of nanoparticles, without blockage of the reactor (Fig. $3 \mathrm{C}$ ). The details of the pilot plant and associated mixer are described in detail elsewhere.
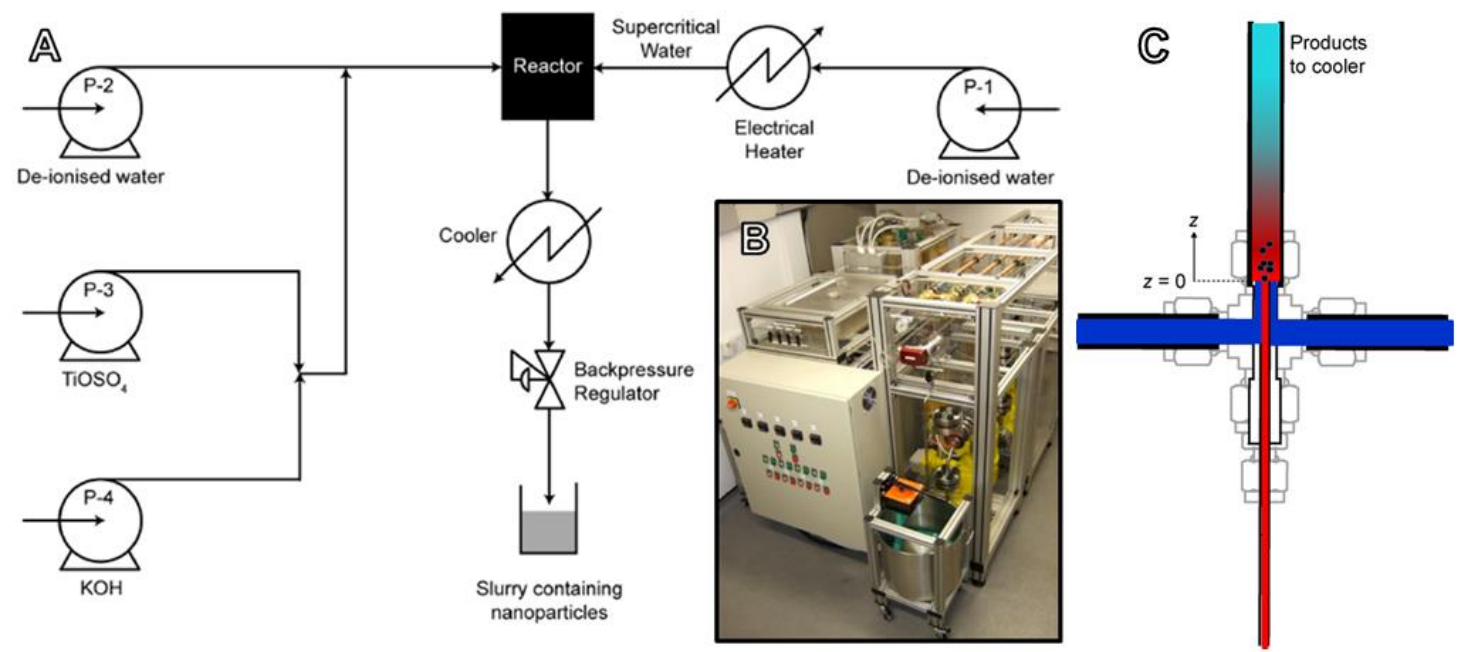

Figure 3 Schematic diagram of the plant configured for producing $6 \mathrm{~nm}$ titania suspensions $(\mathrm{A})$ along with a photograph of the CHFS pilot plant (B) and a diagram of the UCL patented confined jet-mixer (C). 
Table 4 Parameters of titania feedstock synthesis

\begin{tabular}{ccccc}
\hline Precursor & \multicolumn{2}{c}{ Concentration $/ \mathbf{~ m o l ~ d m}^{-3}$} & \multicolumn{2}{c}{ Flow Rate $/ \mathbf{~ m L ~ m i n}^{-1}$} \\
& $\mathbf{6} \mathbf{~ n m}$ & $\mathbf{1 2} \mathbf{~ n m}$ & $\mathbf{6} \mathbf{~ n m}$ & $\mathbf{1 2} \mathbf{~ n m}$ \\
\hline $\mathrm{TiOSO}_{4}$ & 1.0 & 1.0 & 185 & 200 \\
$\mathrm{KOH}$ & 2.0 & 2.35 & 185 & 119 \\
DI Water Feed 1 & - & - & 400 & 400 \\
DI Water Feed 2 & - & - & 30 & 81 \\
\hline
\end{tabular}

The overall process can be described as the reaction of a titanium containing precursor in the presence of base and supercritical water (Eq. 1)

$$
\mathrm{TiOSO}_{4}+\mathbf{m K O H}+(1-\mathbf{m}) \mathrm{H}_{2} \mathrm{O} \rightarrow \mathrm{TiO}_{2}+\left(\frac{\mathbf{m}}{2}\right) \mathrm{K}_{2} \mathrm{SO}_{4}+\left(1-\frac{\mathbf{m}}{2}\right) \mathbf{H}_{2} \mathbf{S O}_{4}
$$

Equation 1 The overall reaction for the production of titiaia by CHFS.

The nanoparticles produced were collected from the CHFS pilot plant as a ca. 1 wt $\%$ suspension, appearing as a milky white liquid. A concentration process was applied to allow ease of transport of materials between experimentation sites. Sodium chloride was added until the suspension separated and transferred to a dialysis tube to clean the supernatant. The resulting material was then concentrated by centrifugation to ca. $25 \mathrm{wt} \%$ and stored until required. The concentrated slurries were then diluted prior to spraying with deionized water to give aqueous titania suspensions of ca. $5 \mathrm{wt} \%$. Typically in suspension spray feedstocks of $10-20 \mathrm{wt} \%$ are used, however, in this instance dilute suspension were selected to observe the effect on HVSFS, a deposition process where excessive phase transformation typically occurs. Suspensions produced by CHFS also require significant de-watering to be used as spray feedstocks therefore dilute suspensions were selected to minimise this requirement. The feedstock suspensions were semi-stable and readily re-dispersible.

All substrates used were $25 \times 25 \times 0.5 \mathrm{~mm}$ AISI 304 stainless steel plates, which had been etched for ca. 30 seconds to a finish of $202 \pm 12 \mathrm{~nm}$ Ra with a ferric acid solution.

\subsection{Characterisation Methods}

X-ray diffraction (XRD) was performed using a Bruker AXS D8 (GADDS) diffractometer, using a Cu Ka X-ray source. Phase composition was estimated by integrating and comparing the anatase (101) and rutile (110) peaks (Eq 1):

$$
C_{A}=\frac{8 I_{A}}{8 I_{A}+13 I_{R}}
$$

Equation 2 Equation for the determination of anatase phase composition percentage where $I_{A}$ and $I_{R}$ are the anatase (101) and rutile (110) relative peak intensities.[8]

Scanning electron microscopy (SEM) was performed using a LEO 1550 Field emission scanning electron microscope (FESEM). For cross-sectional SEM imaging, samples were mounted by vacuum impregnating them with epoxy resin containing conductive filler, before metallographic preparation. Micro-Raman spectroscopy was performed using a Renishaw inVia ${ }^{\text {TM }}$ Raman microscope, measuring in the range 100 to $1000 \mathrm{~cm}^{-1}$. UV-Vis spectroscopy was performed using a Lambda $950 \mathrm{UV} / \mathrm{Vis} / \mathrm{NIR}$ spectrophotometer in reflectance mode in the range 200 to $1500 \mathrm{~nm}$. Surface profilometry was performed using an Alicona Infinite Focus SL, using a 50x objective lens. Selection of $\lambda_{c}$ filters was carried out in agreement with ISO 4288:1998.[9] Photocatalytic activity testing was performed using a sacrificial hydrogen generation test. Prior to testing, the backs of all samples were sputter coated with platinum. The photoactivity of the coating was measured by observing the rate of hydrogen production in a sacrificial solution of $0.1 \mathrm{M} \mathrm{HCl}$ in ethanol and water (1:1).[10-11] The solution was irradiated with two $8 \mathrm{~W} 365 \mathrm{~nm}$ UV lamps at a distance of $8 \mathrm{~cm}$. The gas produced was analysed in a Varian CP-3800 gas chromatograph every 15 minutes for a total of 120 minutes, to determine hydrogen production rate.

All of the coatings ranged in colour from light grey to dark blue, Fig. 4. This change in colour, from the white of the initial feed material, appeared to be as a result of $\mathrm{Ti}^{3+}$ generation. Coatings produced by SFS were the darkest in 
colour, comparable to the SPS coatings produced in the $\mathrm{Ar} / \mathrm{H}_{2}$ plasma at the shortest stand-off distance. SPS Ar/ $\mathrm{H}_{2}$ coatings varied in colour with stand-off, becoming lighter with increasing distance (Fig. 4). This would suggest a level of reoxidation of particles at extended stand-offs. Coatings produced by HVSFS were the lightest in colour, indicating the lowest concentration of $\mathrm{Ti}^{3+}$. $\mathrm{Ti}^{3+}$ gives rise to intermediate electronic states, which allow absorption of visible light (hence the colouration of the coatings), which can result in enhanced photoactivity.[12] However, Ti ${ }^{3+}$ defects can also act as recombination centres, decreasing photoactivity.[13]

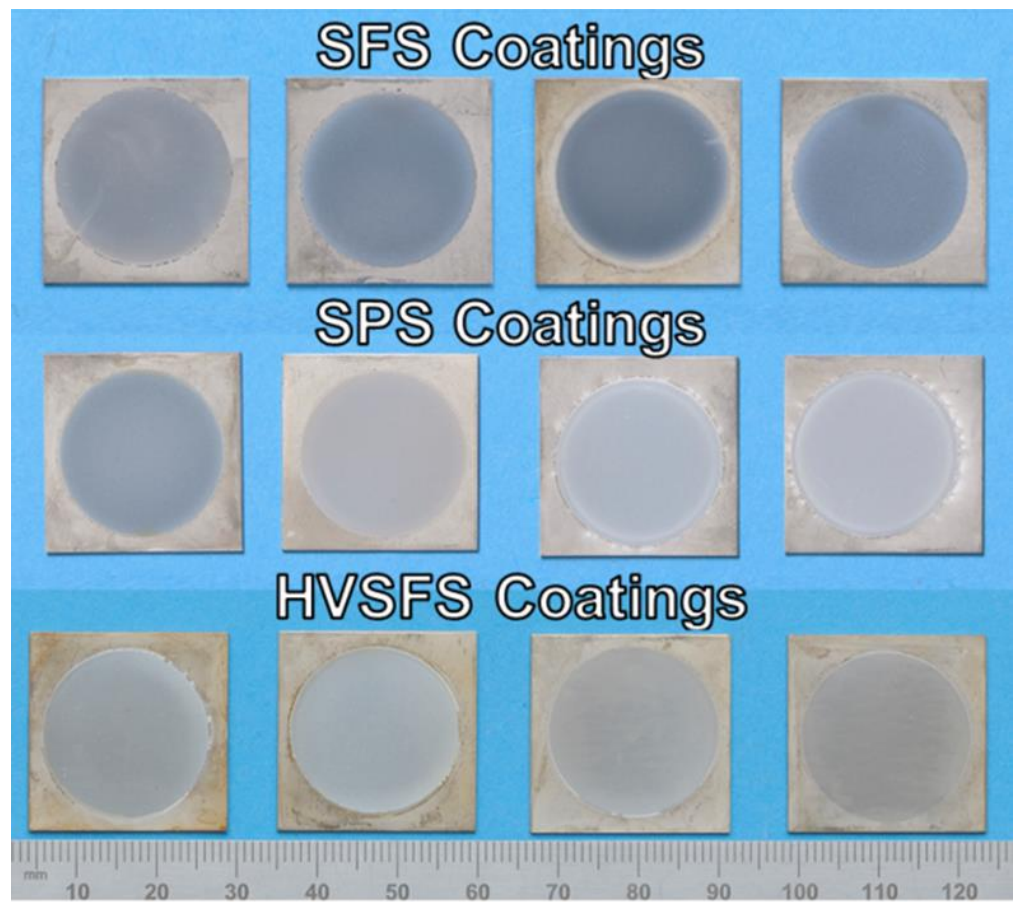

Figure 4 Examples of titania coatings produced by SFS (increasing number passes 5-20), SPS (produced in an Ar/ $\mathrm{H}_{2} \mathrm{plasma}$ with increasing stand-off 55-85mm) and HVSFS (with increasing stand-off 100-130mm).

A tape test, a method commonly used to determine the adhesive qualities of thin films, was performed on all coatings. SFS and SPS coatings were found to be adherent in all cases, while HVSFS coatings varried between conditions, with failures occurring in a number of instances and with no discernible trend. Typically adhesion strength would be assessed by a bond pull test as outlined in ASTM C633-13 (Standard Test Method for Adhesion or Cohesion Strength of Thermal Spray Coatings), however, this was not used due to the low thickness (<50 $\mu \mathrm{m})$ of the coatings, which could have resulted in glue penetration, invalidating results.[14] Thin coatings such as these are desirable for photocatalytic applications as increasing electron-hole recombination can occur with increasing coating thickness.

Table 5 Phase composition ranges for the three suspension spray techniques.

\begin{tabular}{cc}
\hline Coating technique & $\begin{array}{c}\text { Anatase phase } \\
\text { composition / \% }\end{array}$ \\
\hline SFS & $75-95$ \\
SPS & $90-100^{*}$ \\
HVSFS & $100^{*}$ \\
\hline
\end{tabular}




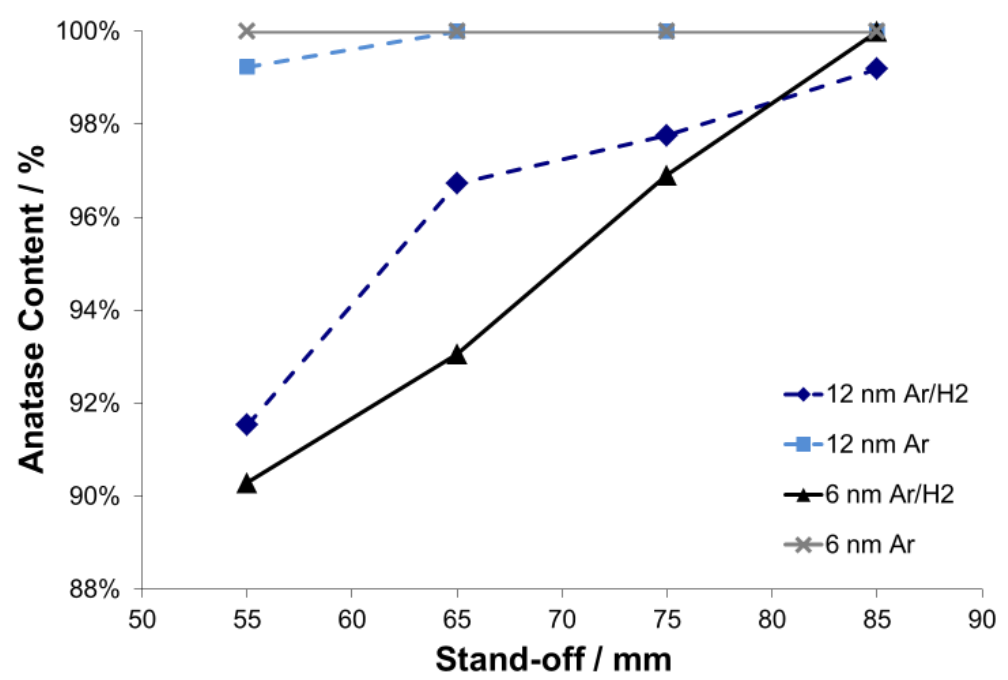

Figure 5 The effect of stand-off distance on anatase phase content of SPS titania coatings.

Phase composition of the coatings was dependent on particle velocity and jet temperature, which affected the melting and resolidification pathways of the titania (Table 5). In SFS, the low particle velocity provided a long dwell time in the flame allowing for significant melting. However, due to the short stand-off distance required (due to the low particle momentum), melting was still limited, showing increased phase transformation with increased stand-off distance (75-95\% anatase). This contrasted with the SPS and HVSFS coatings. SPS trials were in agreement with published work by Jaworski et al., with increasing stand-off distance leading to increased anatase content (90$100 \%$ anatase) due to homogeneous nucleation of anatase from molten titania inflight (Fig 5).[15] This was as a result of the high plasma temperature causing rapid melting of the titania material and allowing changes in resolidification to occur by altering stand-off distance. The effect of stand-off on phase composition was only observed for coatings produced in the $\mathrm{Ar} / \mathrm{H}_{2}$ plasma. This was due to the difference in enthalpy between these two plasmas with the $\mathrm{Ar} / \mathrm{H}_{2}$ resulting in much greater melting of the titania and therefore a greater proportion of the coating was effected by changes in stand-off and resolidification pathway. In both SPS and SFS, phase composition was controlled by altering stand-off distance. Phase composition could not be manipulated with standoff distance in HVSFS trials, in agreement with recent results reported by Toma et al.[16] Herein, this was due to the high particle velocity, allowing minimal time for the particles to melt. The high water content of the suspensions reduced heating of the solids by removing a greater portion of the flame's energy as well as shielding the particles in the hottest area of the flame. This resulted in a phase composition of ca. $100 \%$ anatase for all the HVSFS coatings deposited (with trace rutile observed by micro-Raman spectroscopy). This is significantly greater than is typically observed in HVSFS, with coatings typically in the range of $30-70 \%$ anatase phase. However, this is also outside the often quoted optimal $80 \%$ anatase phase, used in materials such as P25.[17] Despite the apparent presence $\mathrm{Ti}^{3+}$ in the coatings no other titanium oxide compounds (i.e. $\mathrm{Ti}_{2} \mathrm{O}_{3}$ ), were detected in XRD patterns. For observation of $\mathrm{Ti}^{3+}$ concentrations, other methods such as XANES would be required.

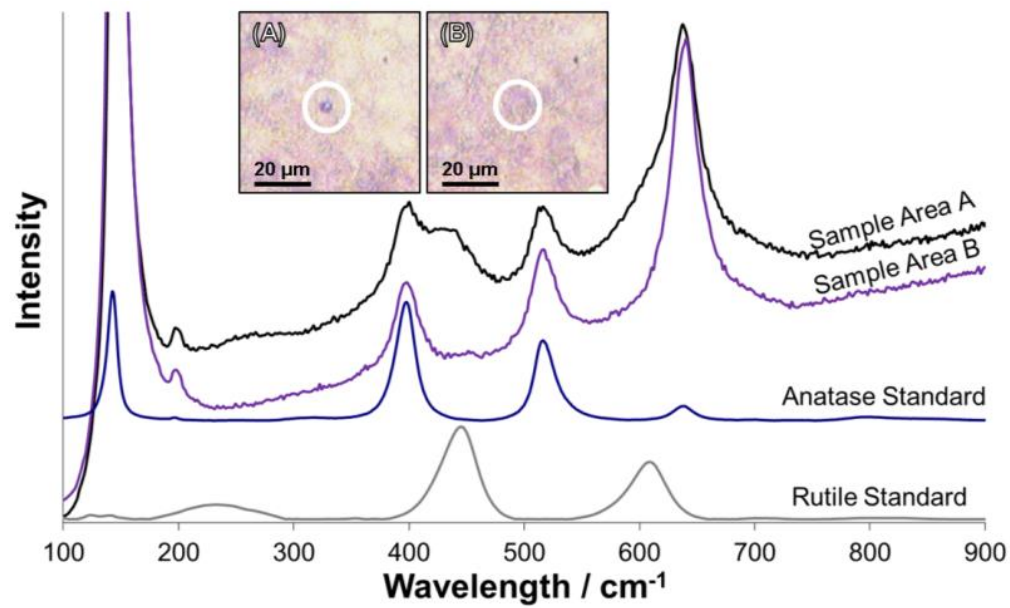

Figure 6 Raman spectra of two areas (sample area A \& B) of a SPS titania coatings produced in an Ar plasma at $65 \mathrm{~mm}$ standoff distance, with reference spectra. The circles on images A and B correspond to the analysed areas.

To observe the phase distribution of the coatings, micro-Raman spectroscopy was used. All coatings were found to be largely composed of anatase phase. Rutile phase was localised in small areas, ca. 1-5 $\mu \mathrm{m}$ in size, varying in concentration between the processes in agreement with XRD data (Fig. 6). These areas were observed as 
blue/black spherical structures (Fig. 6A). Structures such as these have been previously identified as deformed surface quenched agglomerates. These structures were also, intermittently, found to contain solely anatase phase. In these instances these structures would appeared to correspond the agglomerates which had resolidified inflight, a process which favour anatase formation, rather than those which have resolidified on the surface. Micro-Raman analysis also highlighted traces of rutile phase in coatings where only anatase had been detected using XRD, such as all coatings produced by HVSFS and in SPS coatings where an Ar plasma was used coatings.
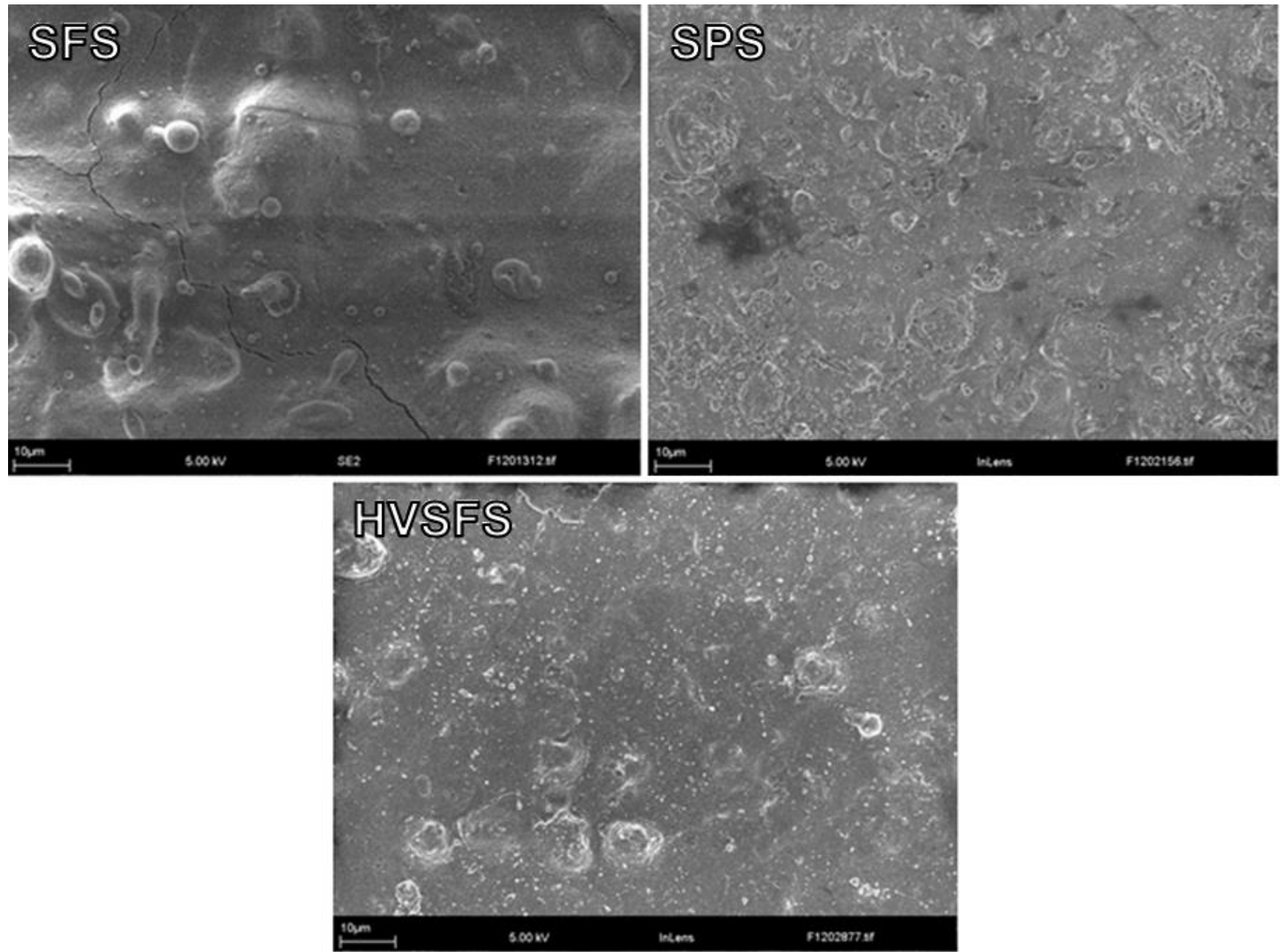

Figure 7 SEM images of SFS, SPS and HVSFS coating surfaces showing the difference in deformed quenched titania.

Under SEM observation, the most significant difference between coatings produced by the three approaches, was the quantity and appearance of deformed surface quenched titania, Fig. 7-8. The quantity of deformed material visible in the coatings decreased from SFS to SPS to HVSFS. This correlated with the phase composition of the coatings as this melted material appeared to be largely rutile (determined by micro-Raman). This change in deformed material concentration was due to the jet velocity and temperature as described above, with SFS having the slowest particle velocity and therefore allowing significant time for particle heating/melting, whereas, the high velocity of the HVSFS jet resulted in more limited melting. The size of the quenched material also decreased from SFS to SPS to HVSFS, due to the increase in velocity/jet turbulence which disrupted agglomeration. This may have also contributed to the minimal quantity of rutile phase in the HVSFS coatings. The small molten droplets could not retain heat for long and underwent homogenous nucleation of anatase phase inflight. Beyond the differences in quenched material, all coatings produced were dominated by the same fine granular zones similar to that of the feedstocks (Fig. 9). This nanostructure was thought to be beneficial for photocatalytic activity by providing greater surface area for absorption. 

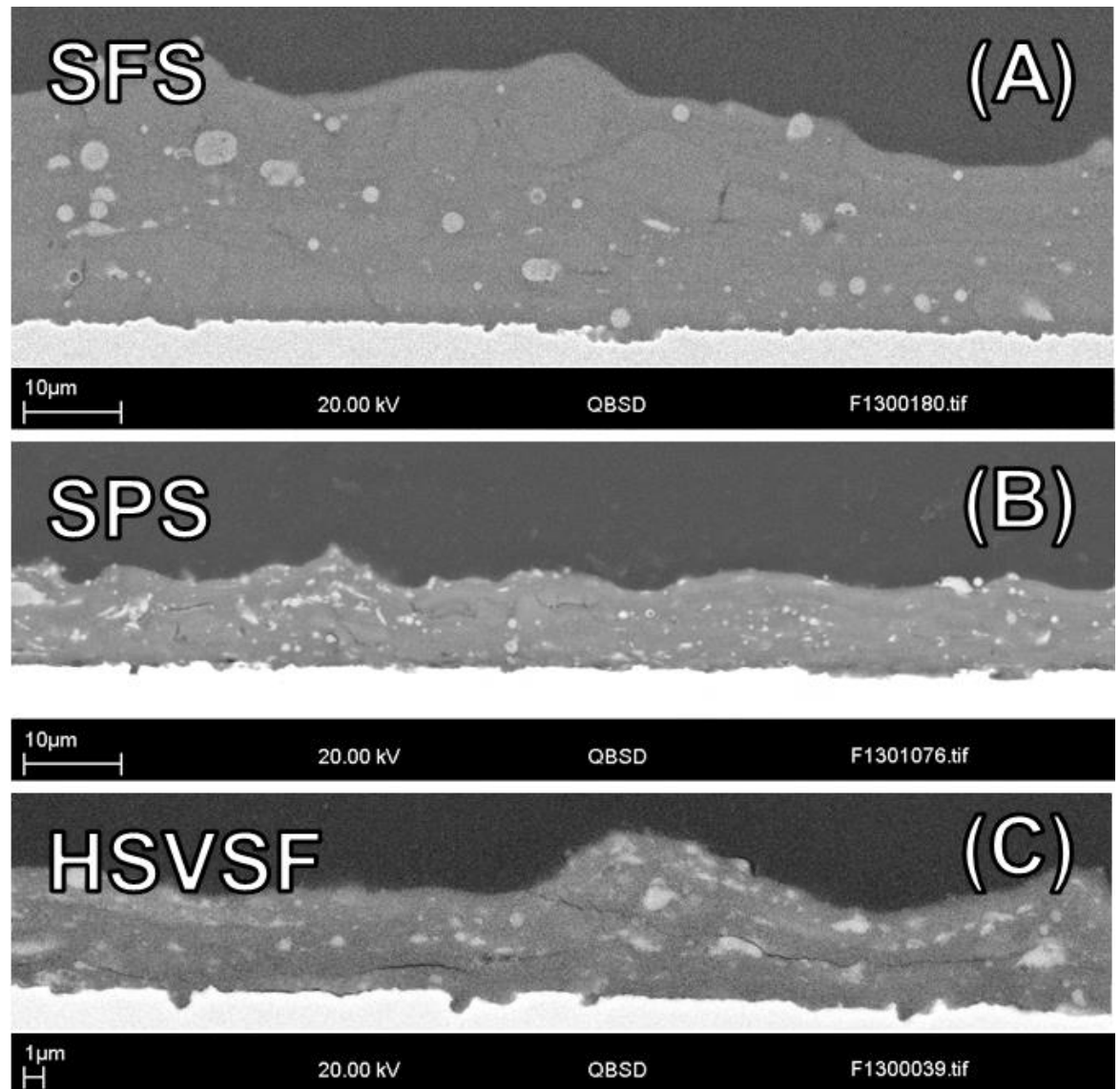

Figure 8 Example cross sections of titania coatings produced by SFS, SPS and HVSFS.
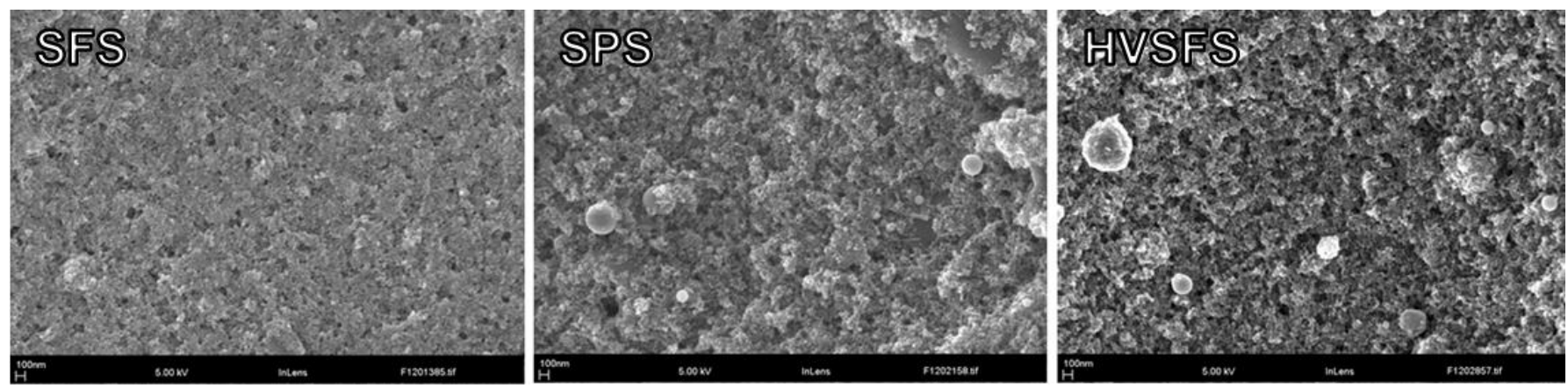

Figure 9 High magnification images of titania coatings produced by SFS, SPS and HVSFS techniques showing the nanostructuring.

Dome like micro-features were also observed in SFS and HVSFS coatings (Fig. 10). This was attributed to a templating effect over the etched substrate. To improve coating adhesion, substrates were etched resulting in groves around the individual grains of the steel substrate surface. These groves appeared to result in preferential deposition of material into dome like structures, ca. 10-20 $\mu \mathrm{m}$ in size. These structures were more clearly expressed with increasing coating mass, observed as a change in surface roughness (Figure 11). No such process was observed for SPS coatings. Dome like microfeatures have previously been observed in YSZ coatings produced by SPS, however, in those instance the structures were attributed to stacking faults resulting in a deliberately porous, columnar structure. No such defects were observed in the coating described herein. 

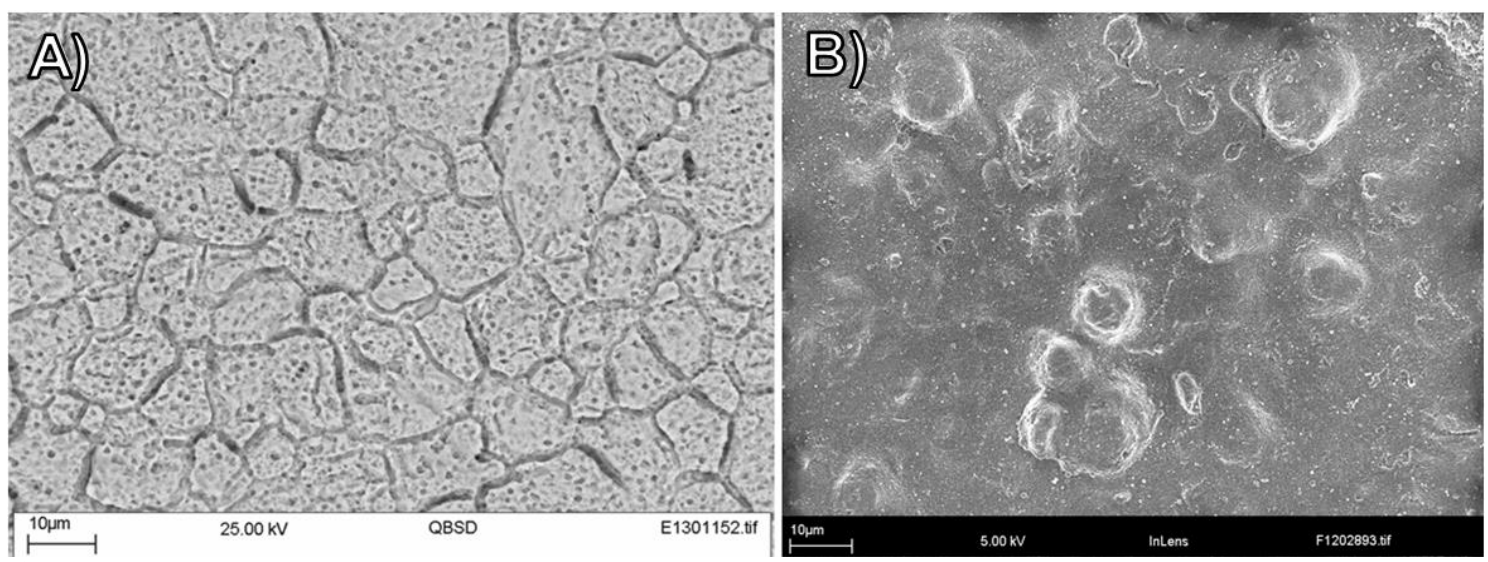

Figure 10 SEM images of an etched steel substrate used (a) and the dome-like surface structures that form as a result of templating over the grains in a HVSFS coating.

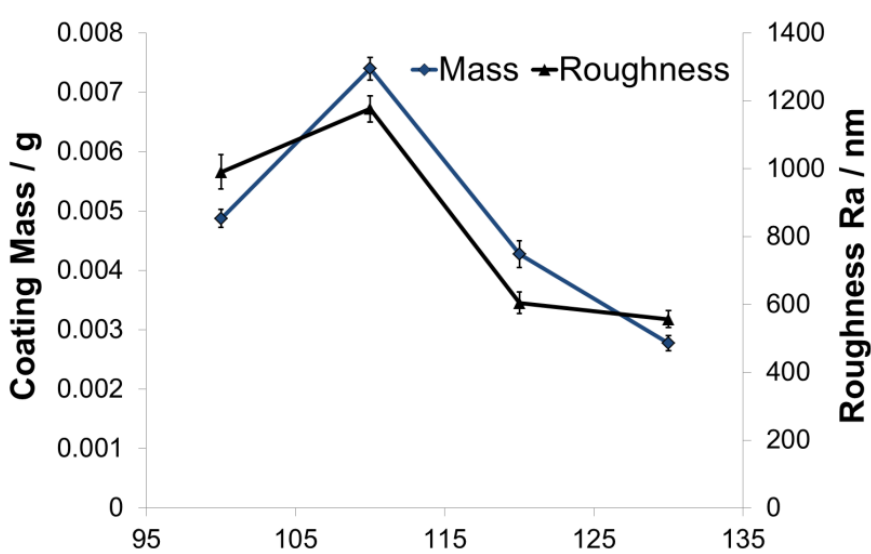

a)

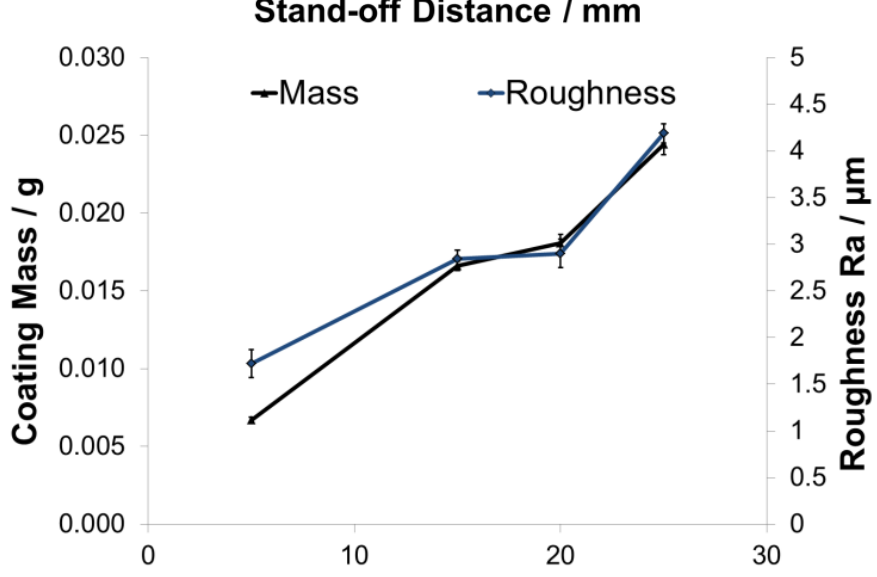

b)

Number of Passes

Fig. 11. The correlation between coatings mass and roughness (Ra), for $6 \mathrm{~nm}$ HVSFS coatings (a) and $12 \mathrm{~nm}$ SFS coatings produced at $45 \mathrm{~mm}$ stand-off distance.

Roughness has been found to be an important factor contributing to coating photoactivity, with larger Ra (average roughness) values resulting in greater photoactivities, in some cases. Ra was therefore considered when comparing these techniques. SPS produced the roughest coatings ( $R$ a 2.4-12.3 $\pm 0.4 \mu \mathrm{m}$ ) followed by SFS (Ra 1.7$5.6 \pm 0.3 \mu \mathrm{m})$ and then HVSFS (Ra 0.6-1.2 $\pm 0.1 \mu \mathrm{m})$. This difference in coating roughness was attributed to the difference in mass of the coatings produced by the three processes (HVSFS coatings having the lowest mass). The different turbulences of the jets may also play a significant role with greater turbulence resulting in small agglomerate features. However, the reason for the high roughness values for the SPS coatings is not known. 


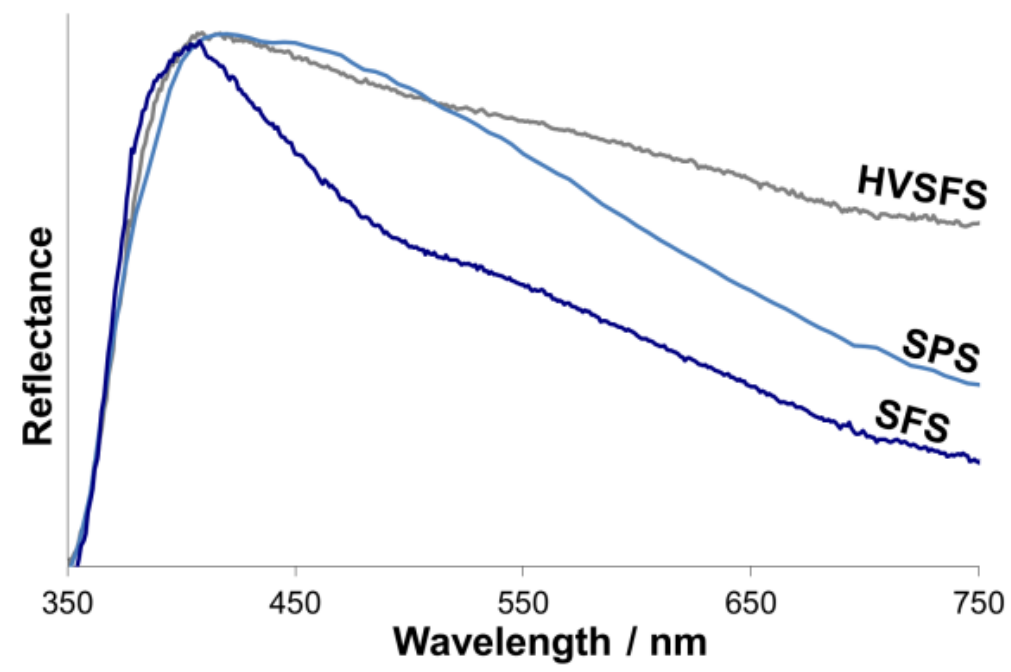

Fig. 12. UV-Vis spectra for SFS, SPS and HVSFS coatings with maximum visible light absorbance.

Due to the differing temperature and velocity profiles of the three spray processes and their effect on phase composition, crystal size and $\mathrm{Ti}^{3+}$ concentration, UV-Vis spectroscopy was performed. SFS gave coatings with the darkest blue colouration, followed by SPS and HVSFS (least dark). This was observed via UV-Vis spectroscopy as a significant absorbance in the visible region $(>450 \mathrm{~nm})$ which decreased in intensity from SFS to SPS to HVSFS, Fig 12. This trend in concentration of $\mathrm{Ti}^{3+}$ was attributed to the relative velocities of the processes, with SFS providing the greatest inflight time for reduction reactions with combustion products (or hydrogen in the case of SPS) followed by SPS and HVSFS. Titania can also lose oxygen at temperatures of $>500{ }^{\circ} \mathrm{C}$, which is well within the temperature ranges reached during these processes.[18] As has been previously observed from SEM images, SFS coatings also underwent the greatest heating, with the greatest percentage of melted material, followed by SPS. This difference in particle heating was a result of the differing particle velocities of the three processes and also contributed to the differences in $\mathrm{Ti}^{3+}$ and visible light absorbencies of the coatings. A reduction in visible light absorbance was observed at increased stand-off distances in the case of SPS $\mathrm{Ar} / \mathrm{H}_{2}$ and HVSFS coatings, suggesting some reoxidation of particles with extended flight times. No significant difference in band-gap was observed between the coatings produced by the three processes. All coatings had band-gaps in the range $\mathrm{ca}$. 3.0 to $3.3 \mathrm{eV}$, typical for mixed phase titania (rutile $3.0 \mathrm{eV}$ and anatase $3.2 \mathrm{eV}$ ).[19]

Photoactivity of SFS, SPS and HVSFS coatings was determined by a sacrificial hydrogen generation test. Samples were compared with compressed disks of P25, the highly photoactive titania material often used as a standard in photocatalytic testing, as reported by Elouali et al. [10] Samples were also compared to CVD titania coatings, previously reported by Hyett et al., CVD being a competing coating method for the production of thin photoactive coatings.[11] These two sets of results could not be compared with each other due to the differing ways in which they were calculated and reported in their respective publications. In both definitions of maximum hydrogen production rate $\left(\mu \mathrm{molhr}{ }^{-1} \mathrm{~cm}^{-2}\right.$ and $\left.\mu \mathrm{molhr}^{-1} \mathrm{~g}^{-1} \mathrm{~cm}^{-2}\right)$ the order of photoactivity was SFS>SPS>HVSFS, Fig. 13. This was attributed to a number of factors. Whilst there is still discussion as to the effect of phase composition on photoactivity and the optimal composition thereof (largely thought to be $80 \%$ anatase $20 \%$ rutile), a consensus is generally held that the presence of rutile significantly increases coating photoactivity.[20] This is ascribed to vectorial charge separation when the phases are in contact. It could be due to this that SFS coatings were more active than SPS and HVSFS coatings. The maximum hydrogen generation achieved by SFS was from a coating with ca. $80 \%$ anatase while the maximum achieved by SPS and HVSFS was for a coating containing only trace rutile. The highest hydrogen generation attained by an SPS coating was for a ca. $100 \%$ anatase coating, however, this was likely due to the enhanced recombination effects in SPS coatings containing rutile phase as a result of the presence of $\mathrm{Ti}^{3+}$ (caused by the use of $\mathrm{Ar} / \mathrm{H}_{2}$ plasma relative to $\mathrm{Ar}$ plasma).[1] SPS coatings containing $90 \%$ anatase $10 \%$ rutile were produced in an $\mathrm{Ar} / \mathrm{H}_{2}$ which led to a colour change (and assumed titania reduction) comparable to that of the SFS coatings. SPS coating activity was found to be greater than that of HVSFS coatings, this was despite the fact that the coatings with the greatest activity in both instances had ca. $100 \%$ anatase phase composition with trace rutile. This difference in $\mathrm{H}_{2}$ generation may have been as a result of the different surface roughness of coatings produced by SPS and HVSFS processes $(6.1 \pm 0.1 \mu \mathrm{m}$ and $1.0 \pm 0.1 \mu \mathrm{m}$, respectively), with the greater roughness of the SPS coatings providing greater surface area for absorbance. From this it was clear that assigning the trend in photocatalytic activity to a single property of the coatings was not possible. Rather, coating activity has been observed to be affected by a number of factors including phase composition, $\mathrm{TiO}_{x}$ concentration and surface roughness/area in these results alone, with more reported in the literature.[24] A challenge in suspension spray is therefore, to observe the effect of each of these aspects separately, with spray parameters having far reaching effects on coating properties. Similar observations of more complex coating parameters affecting coating activity have been reported previously.[21] Testing in this instance was also 
performed under UV illumination. To gain a greater understanding of the effect of the visible light absorbance of suspension spray coatings further testing should be performed using a solar simulator.
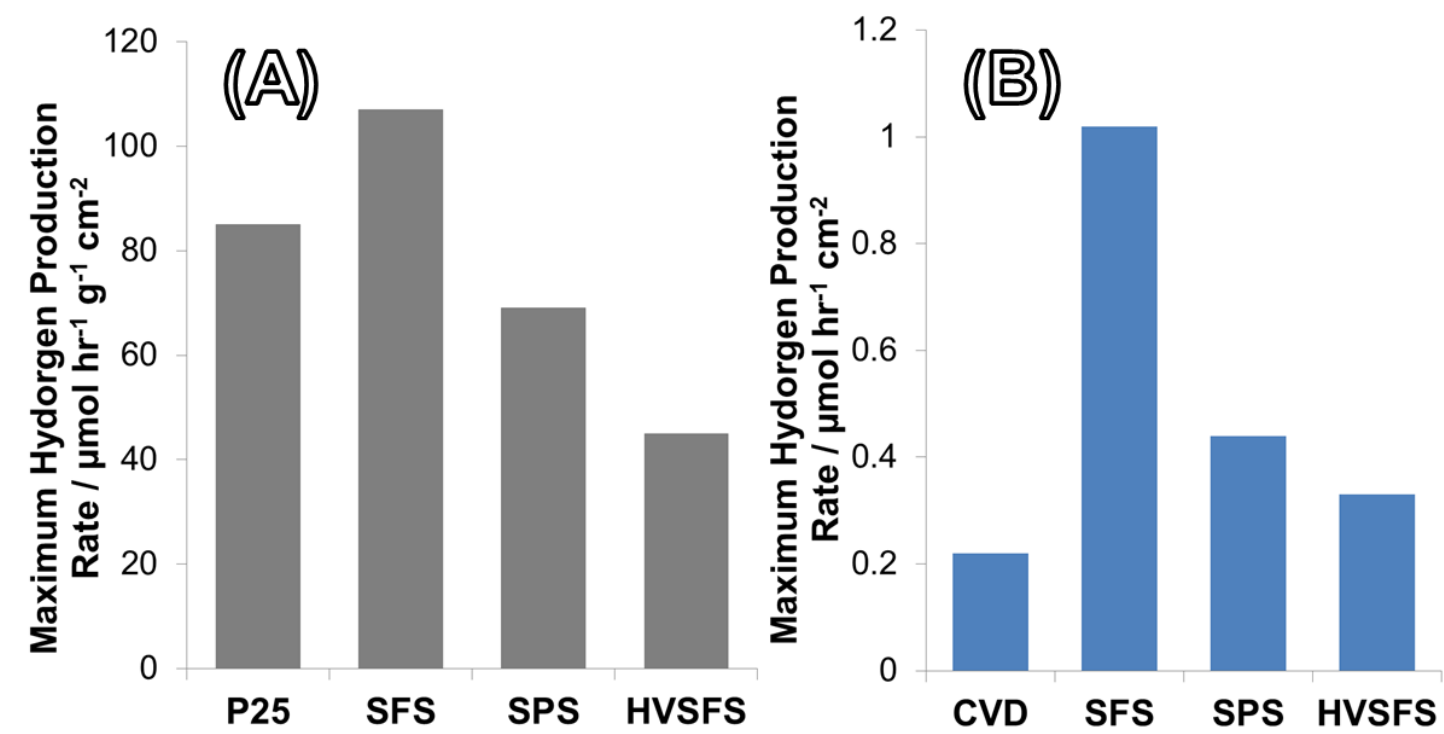

Fig. 13. Graph showing the maximum hydrogen production rates of SFS, SPS and HVSFS titania coatings relative to a sintered disks of P25 (A) and rutile CVD coatings (B).

When comparing SFS, SPS and HVSFS coatings with compressed disks of P25, only SFS was observed to have a greater activity, Fig. 13A. Maximum hydrogen generation for the SFS process was observed to be $107 \mu \mathrm{molhr}^{-1} \mathrm{~g}$ ${ }^{1} \mathrm{~cm}^{-2}$ relative to the $85 \mu \mathrm{molhr}^{-1} \mathrm{~g}^{-1} \mathrm{~cm}^{-2}$ for P25 discs, an increase of ca. 26\%.[11] This demonstrates the high photoactivity of SFS coatings and suggests that SFS is a potential route to the production of photoactive coatings for applications such as water purification. When comparing suspension spray coatings produced herein with CVD analogues, SFS, SPS and HVSFS were all more active, producing up to 5 times more hydrogen in the case of SFS, Fig. 13B.[11] This demonstrated the potential of suspension spray techniques, particularly SFS, for the production of photoactive titania coatings relative to existing methods. It is also noted that suspension spray is a much more scalable technique than methods such as CVD and PVD, due to their use of expensive deposition or vacuum equipment. However, it should also be said that CVD coatings used for this comparison were composed of rutile phase and would therefore have decreased activity relative to an anatase alternative.

\section{$4 \quad$ Scale-up, Cost and Technical Challenges}

Suspension spray facilities are a combination of nano-suspension feeding equipment and conventional thermal spray torches (although some custom suspension spray torches are now commercially available). When comparing the scalability and expense of the suspension spray processes, the feeding equipment was ignored as this would not change significantly between torches in an external injection setup common for photocatalytic titania coatings and only the torch and controller systems were considered. Flame spray is the oldest and simplest of the thermal spray techniques. The capital and operational costs of flame spray are also significantly less than for plasma spray or HVOF systems.[22] Flame spray also requires less training and experience to operate safely and effectively.

All suspension spray methods are typically carried out at a shorter stand-off distance than their thermal spray counter parts (e.g. HVSFS $100 \mathrm{~mm}$, HVOF $200 \mathrm{~mm}$ ).[23] As such, there is an increased thermal load on the substrate and jigging (Fig. 14). Here, this issue was resolved by the use of a custom designed water cooled jig. However, this would be impractical in a large scale, production scenario. Other researchers have managed substrate temperature through the use of proprietary gas cooling technology.[24] As such, it is clear that thermal loading is a significant challenge in suspension spraying. Of the three processes, SFS, while having the shortest stand-off distance, transfers the least heat into the substrate/jigging due to the lower temperature and kinetic force of the jet relative to SPS and HVSFS respectively. SFS therefore presents fewer difficulties as regards thermal management. 


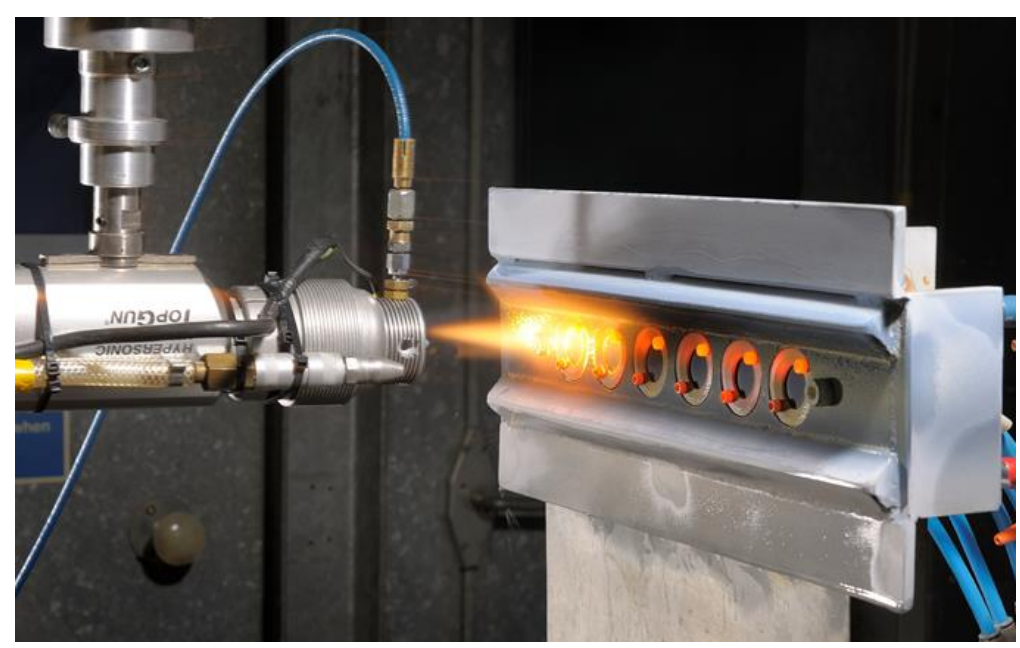

Figure 14 Photograph showing the large thermal load experienced by the substrate and jigging from the passing HVSFS torch as seen by the glowing mounting rings. This photograph is taken from related trials using alcoholic titania suspensions with comparable flame conditions.

SFS is the least common of the suspension spray techniques, with no commercial SFS equipment currently available and a decreased literature base relative to SPS and HVSFS. Suspension plasma spray particularly has gained significant attention for the deposition of improved YSZ thermal barrier coatings.[25] As such, SFS has the drawback that it may not have the same support and supply network as SPS and HVSFS in the future.

The most significant practical problem with suspension spray is blockages. In a production setting, blockages can be costly and time consuming.[26] However, during spray trials, no one individual process resulted in a greater level of observed blockages. Blockages in suspension spray are also becoming more manageable with the introduction of commercial suspension feeding equipment such as the Mettech NanoFeed ${ }^{\mathrm{TM}}$.

Considering all of the above issues, SFS appears to have a number of desirable characteristics compared with SPS and HVSFS, namely its relative simplicity, low expense and decreased substrate thermal load.

\section{4}

\section{Conclusion}

Coatings were deposited via SFS, SPS and HVSFS techniques, characterised and their photocatalytic capabilities evaluated. Suspension flame spray was able to produce phase controlled nanostructured coatings, as previously also reported for SPS.[1] SFS and SPS coatings were found to be adherent using a tape test, however failures were noted for HVSFS coatings. SFS coatings were shown to be up to $32 \%$ more photoactive in a hydrogen generation test than those produced by SPS. SFS coating activity also compared favourably with analogue samples produced from conventional materials (P25) and processes (CVD). However, due to the large number of characteristics which can influence photoactivity of suspension spray coatings, SFS should only be highlighted as a potential method for improved photoactivity. SFS is a far more simple technique, its capital and operational cost are significant less and it also demonstrates a decreased tendency for substrate overheating. However, it should be highlighted that far less is currently known about SFS titania coatings and the possible associated technical challenges and that further research is required in this area.

Acknowledgements

This research was partially funded by the UK's Technology Strategy Board (TSB) and the Engineering and Physical Sciences Research Council (EPSRC) under Project 100898 "Nanocrystalline Water Splitting Photodiodes II; Device Engineering, Integration \& Scale-up". The associated ESPRC EngDoc sponsorship for B Robinson was co-funded by TWI Limited under UCL's Industrial Doctorate Centre.

\section{6}

\section{References}

[1] Robinson, B.W., et al., Suspension plasma sprayed coatings using dilute hydrothermally produced titania feedstocks for photocatalytic applications. Journal of Materials Chemistry A (2015) 3, 24, pp. 12680/12689

[2] Liu, Y.X., et al., Deposition, Characterization, and Enhanced Adherence of Escherichia coli Bacteria on FlameSprayed Photocatalytic Titania-Hydroxyapatite Coatings. Journal of Thermal Spray Technology (2013), 22, 6, pp. 1053/1062.

[3] Bolelli, G., et al., Properties of High Velocity Suspension Flame Sprayed (HVSFS) $\mathrm{TiO}_{2}$ coatings. Surface \& Coatings Technology (2009), 203, pp. 1722/1732. 
[4] Oksa, M., et al., Optimization and Characterization of High Velocity Oxy-fuel Sprayed Coatings: Techniques, Materials, and Applications. Coatings (2011), 1, pp. 17/52.

[5] Toma, F.L., et al., Microstructures and Functional Properties of $\mathrm{Al}_{2} \mathrm{O}_{3}$ and $\mathrm{TiO}_{2}$ Suspension Sprayed Coating: An Overview. Thermal Spray 2009: Proceedings of the International Thermal Spray Conference, Marple, B. R., et al. (2009), pp. 168/173.

[6] Toma, F.L., et al., Comparative study on the photocatalytic behaviour of titanium oxide thermal sprayed coatings from powders and suspensions. Surface \& Coatings Technology (2009), 203, 15, pp. 2150/2156.

[7] Zhang, Z., et al., Direct continuous hydrothermal synthesis of high surface area nanosized titania. Journal of Alloys and Compounds (2009), 476, 1, pp. 451/456.

[8] N. Berger-Keller, et al., Microstructure of Plasma-Sprayed Titania Coatings Deposited from Spray-Dried Powder. Surface Coatings Technology (2007), 168, pp. 281/290

[9] BS EN ISO 4288:1998 Geometric product specification (GPS). Surface texture. Profile method: Rules and procedures for the assessment of surface texture. 1998, BSI.

[10] Elouali, S., et al., Photocatalytic evolution of hydrogen and oxygen from ceramic wafers of commercial titanias. Journal of Photochemistry and Photobiology a-Chemistry. (2010), 216, 23, pp. 110/114.

[11] Hyett, G., et al., Substrate-Dependant Ability of Titanium (IV) Oxide Photocatalytic Thin Films Prepared by Thermal CVD to Generate Hydrogen Gas from a Sacrificial Reaction. Chemical Vapor Deposition. (2010), 16, 1012, pp. 301/304.

[12] Saputera, W.H., et al., Ti3 ${ }^{3+}-$ containing titania: Synthesis tactics and photocatalytic performance. Catalysis Today. (2015), 246, pp. 60/66.

[13] Robinson, B.W., et al., The Effect of Fuel Ratio on Photoactivity of Suspension Flame Sprayed Coatings. ITSC 2015, May 11-14, Long Beach, CA.

[14]ASTM C633-13, in Standard Test Method for Adhesion or Cohesion Strength of Thermal Spray Coatings. 2013.

[15] Jaworski, R., et al., Influence of suspension plasma spraying process parameters on $\mathrm{TiO}_{2}$ coatings microstructure. Journal of Thermal Spray Technology. (2008), 17, 1, pp. 73/81

[16] Toma, F.L., et al., Parameters Influencing the Photocatalytic Activity of Suspension-Sprayed $\mathrm{TiO}_{2}$ Coatings. Journal of Thermal Spray Technology, (2014), 23, 7, pp. 1037/1053.

[17] Wu, C.Y., et al., Investigation on the synergetic effect between anatase and rutile nanoparticles in gas-phase photocatalytic oxidations. Catalysis Today. (2004), 93-5, pp. 863/869.

[18] Erne, M., et al., Basic principles of reaching triboactive coatings by mixing of nanosized feedstock powders in the suspension plasma spraying process. Materialwissenschaft Und Werkstofftechnik. (2010). 41, 7, pp. 541/546.

[19] Luttrell, T., et al., Why is anatase a better photocatalyst than rutile? - Model studies on epitaxial $\mathrm{TiO}_{2}$ films. Scientific Reports. (2014), 4, pp. 8.

[20] Li, K., et al., Enhanced Photoactivity of Fe plus N Codoped Anatase-Rutile $\mathrm{TiO}_{2}$ Nanowire Film under Visible Light Irradiation. International Journal of Photoenergy. (2012), pp. 8

[21] Bannier, E., et al., Microstructure and photocatalytic activity of suspension plasma sprayed $\mathrm{TiO}_{2}$ coatings on steel and glass substrates. Surface \& Coatings Technology. (2011), 206, 2-3, pp. 378/386.

[22] Champagne, V.K., The cold spray materials deposition process: Fundamentals and applications. 2007: Elsevier.

[23] Bolelli, G., et al., Deposition of $\mathrm{TiO}_{2}$ Coatings: Comparison between High Velocity Suspension Flame Spraying (HVSFS), Atmospheric Plasma Spraying and HVOF-spraying, Thermal Spray 2009: Expanding Thermal Spray Performance to New Markets and Applications, B.R. Marple, et al. (2009), ASM International: Las Vegas. pp. $207 / 212$

[24] Joulia, A., et al., Tailoring the Spray Conditions for Suspension Plasma Spraying. Journal of Thermal Spray Technology. (2015), 24, 1-2, pp. 24/29.

[25] Schlegel, N., et al., Columnar Structured Thermal Barrier Coatings (TBCs) by Suspension Plasma Spraying (SPS), ITSC 2014. (2014), DSV: Barcelona, Spain.

[26] Toma, F.-L., et al., Suspension Thermal Spraying - The Potential of a New Spray Technology. Thermal Spray Bulletin. (2010), 3, 1, pp. 24/29. 